\title{
Generating Rapid DNA Profiles from Crime Scene Samples Commonly Encountered in the United Arab Emirates
}

\author{
Aamer Alshehhi ${ }^{1,2^{*}}$ and Reena Roy ${ }^{1}$ \\ ${ }^{1}$ The Pennsylvania State University, Forensic Science Program, Eberly College of Science, University Park, PA 16802, UAE \\ ${ }^{2}$ Forensic Evidence Department, Abu Dhabi Police GHQ, UAE
}

"Corresponding author: Aamer Alshehhi, M.P.S, The Pennsylvania State University, Forensic Science Program, Eberly College of Science, University Park, PA 16802, UAE, Tel: +1-202-320-1202; E-mail: aamer.alshehhi@gmail.com

Rec date: April 21, 2015 Acc date: July 25, 2015 Pub date: July 30, 2015

Copyright: @ 2015 Alshehhi A, et al. This is an open-access article distributed under the terms of the Creative Commons Attribution License, which permits unrestricted use, distribution, and reproduction in any medium, provided the original author and source are credited.

\begin{abstract}
Short tandem repeat (STR) DNA profiles are routinely generated from blood and saliva stains found on items of evidence collected at crime scenes. Current study utilized the fully integrated RapidHIT ${ }^{\mathrm{TM}}$ Human DNA Identification System (IntegenX, Pleasanton, CA, USA) to generate STR profiles from simulated crime scene evidence samples, similar to the ones commonly encountered at indoor crime scenes in the United Arab Emirates (UAE). The two body fluids were deposited on each of these items and processed. Data generated from 120 samples using this automated system and the "Run Other Samples" instrument protocol were in complete concordance with the data generated with traditional method used in forensic laboratories. Sensitivity study performed with this instrument indicated that complete profiles can be obtained from $0.25 \mu \mathrm{L}$ of blood and $10.0 \mu \mathrm{L}$ of saliva.
\end{abstract}

Varying amounts of both of the body fluids were deposited on 25 different types of substrates containing potential inhibitors such as dyes and soil. Fifteen of these substrates containing body fluids were incubated at $48^{\circ} \mathrm{C}$ heat and $43 \%$ humidity for 24 hours. These conditions were used to mimic indoor crime scenes at the UAE during the Summer Season. Complete and concordant profiles were generated from most of these samples.

Keywords: Forensic research; RapidHIT ${ }^{\mathrm{rw}}$; STR typing; Crime scene substrates; Weather; United Arab Emirates; PowerPlex $16 \mathrm{HS}$

\section{Introduction}

Currently, short tandem repeat (STR) analysis is routinely performed in forensic laboratories for generating DNA profiles from blood and saliva samples encountered at crime scenes [1-7]. Traditional methods for generating STR profiles include several steps; extraction of DNA from body fluids found on different types of substrates, quantification of DNA in the extracted samples, amplification of optimal amount of DNA using polymerase chain reaction (PCR), separation of the generated DNA fragments using methods such as capillary electrophoresis and finally, the analysis of the generated profiles. Instruments and steps such as the robotic extraction processes help the workflow go faster. Commercially available kits which allow for direct amplification of body fluids deposited on substrates with or without lysing agents, allow for faster turn-around time [8-12]. However, the process still must be performed in a laboratory setting, where the body fluids identified on items of evidence must be extracted in the pre-PCR room. After this step, the amplification and electrophoresis are performed in a separate area commonly referred to as the post-PCR room. These areas are separated in order to minimize contamination. The analysis of the amplified product is then performed using the software the laboratory chooses. Thus, even with the direct amplification of body fluids and the use of robotic instruments, the process remains somewhat labor intensive and may require the analysts to spend much hands-on time. In addition, due to contamination issues with forensic evidence, DNA analysis must be performed in a laboratory where environment is controlled rigidly by the laboratory protocols.

In order to expedite the process, particularly with reference samples, companies such as NetBio and IntegenX have developed instruments, which provide 'Rapid DNA' analysis [13-18]. Usually, these instruments are used for analysis of reference samples such as buccal swabs. The advantage of such an instrument is that only one device is required from start to finish. These automated instruments perform the extraction of DNA, and while there is not a separate step for the quantification of DNA, the bead technology used for extraction normalizes the amount of DNA extracted. Eventually, the PCR amplification using STR primers takes place within the same instrument. The buffer and polymer needed for the electrophoresis are contained in the disposable cartridges. The instrument includes a separate capillary electrophoresis component which allows the separation of fragments and collection of raw data. The files containing the data can then be exported and analyst is able to analyze the resulting STR profiles at a separate workstation. The entire process from start to finish takes approximately 90 minutes. The operator merely loads the entire sample into the disposable cartridge channel or the disposable microfluidic chips provided by the vendors and walks away until the data is generated. DNAscan from NetBio uses microfluidic BioChipSet cassette. Each cassette has an allelic ladder that is run during the sample process. If the allelic ladder fails during the run, a fixed set of sizing bins is used for designation which is built into the Expert System software.

The RapidHIT $^{\mathrm{m}}$ System from IntegenX is an automated device for STR genotyping. It performs all of the above steps in approximately 90 minutes. The cartridges contain the positive and negative controls, as 
well as the ladder in three separate channels. The amplification of the positive and negative controls takes place at the same time as the amplification of up to five unknown samples which are inserted into five independent channels. It is an instrument which performs DNA extraction of body fluids deposited on various types of substrates, and the substrate can be inserted directly into each channel. Each piece of evidence is inserted into one single slot of the cartridge and is processed through individual channels for amplification with PowerPlex ${ }^{\oplus} 16 \mathrm{HS}$ chemistry (Promega Corporation, Madison, WI, USA). The reagents used in the amplification are optimized for this platform. This allows generation of 15 STR loci and the amelogenin gender locus. The 13 core loci used in the CODIS (Combined DNA Index System) as well as two additional loci, Penta D and Penta E [5] are included in the PowerPlex ${ }^{\infty} 16 \mathrm{HS}$ System. Once that step is complete, bead-based normalization process using the DNA IQ $^{\text {in }}$ System (Promega Corporation, Madison, WI, USA) takes place. The DNA IQ ${ }^{\mathrm{m}}$ System controls and limits the maximum quantity of extracted DNA and evidence is processed through an individual channel. No direct quantification of extracted DNA is necessary as the DNA IQ ${ }^{\mathrm{m} x}$ System controls and limits the quantity of extracted DNA necessary for amplification with the primers within the same system. The RapidHIT ${ }^{\text {mox }}$ System contains a component that performs capillary electrophoresis and detects DNA fragments. The amplified products are then analyzed by the instrument using GeneMarker ${ }^{\bullet}$ HID Software from SoftGenetics ${ }^{\star}$ (State College, PA, USA). The reagents, positive and negative controls as well as the ladders are already assembled in the factory-made disposable reagent cartridges.

Finally, the generation of STR profile takes place and analyzed by the analyst. The system is also capable of amplifying STR loci contained in the commercially available kits such as GlobalFiler ${ }^{\text {rex }}$ (Life Technologies, Foster City, CA, USA) and Promega ESI loci. The ESI Fast System is used mainly by the European forensic community at this time.

This portable instrument is sturdy and can be used at places such as a police station or at a crime scene where controlled laboratory environment is not available. This is also an ideal device for running in the laboratory's controlled setting if an evidence sample must be processed quickly for investigation purposes.

The analysis software that comes with the RapidHIT ${ }^{\mathrm{m}}$ DNA Identification System is an integral part of the instrument and it is a hands-free interpretation of the DNA profiles. However, this on-board software gives minimum information through the screen of the instrument or any attached screen. The files of the runs can be transferred using a USB portable drive to another computer or workstation for future analysis with GeneMarker ${ }^{\circledR}$ HID software.

In order to validate the results generated with the RapidHIT ${ }^{\mathrm{m}}$, the same analysis must be performed with standard STR typing methods already practiced in forensic DNA laboratories. The results generated by both of the methods should be compared for concordance and quality of profiles.

The current research evaluates the capability of the RapidHIT DNA Identification System from IntegenX for generating complete and concordant profiles from blood and saliva deposited on simulated evidence samples, such as the ones commonly found at crime scenes in the UAE.

The primary focus of this study was to validate "Run Other Samples" protocol using both blood and saliva. The sensitivity of the automated device was tested using different volumes of these body fluids. Also, the robustness of the system against potential PCR inhibitory factors that might be present in different substrates used in this study was monitored. Additionally, the reliability of the instrument was checked by mimicking weather conditions normally encountered at indoor crime scenes in the UAE Summer Season. In order to test the robustness of the automated system, blood and saliva samples were deposited on various substrates and these substrates were then incubated under the same weather conditions. The accuracy of the system, as well as contamination, if any, between channels in the same cartridge and between consecutive runs on the same instrument was also examined. Finally, the last objective included comparing the results generated with the RapidHIT ${ }^{\text {rax }}$ System with profiles obtained with traditional STR typing method practiced in the authors' laboratories. This included using standard extraction protocol, amplification with the PowerPlex ${ }^{\oplus}$ 16HS amplification kit and fragment analysis by capillary electrophoresis. Duplicate experiments using the same samples and deposited on the same substrates, and subjected to the same conditions were performed in order to determine an expected average value of the amount of input DNA for each sample run with the RapidHIT ${ }^{\mathrm{m}}$ System. Comparison of the quality of the profiles as well as concordance of the results were included as part of this research.

\section{Materials and Methods}

\section{Collection of body fluids}

All samples were collected following the appropriate Institutional Review Board (IRB) and Institutional Biosafety Committee (IBC) guidelines at The Pennsylvania State University. All samples were anonymized. Blood samples were obtained from three deceased individuals (one male and two females). These were designated as M1 (male), F1 and F2 (two females). Blood samples were collected in Vacutainer ${ }^{\oplus}$ tubes containing EDTA (Becton, Dickson and Company; Franklin, NJ, USA).

The saliva samples were received from two living donors. These two samples were designated as Donor 1 (female) and Donor 2 (male). Saliva samples from these two donors were collected as sputum in sterile centrifuge tubes and centrifuged for five minutes to minimize the viscosity and create a homogenous solution for pipetting. After discarding the viscous layer the homogenous saliva solution was used for experiments. Moreover, both donors were asked to either, put the same objects inside the mouth, lick or spit on some other, and chew some of the substrates in order to deposit their cells or saliva in a similar manner to mimic the crime scene evidence. Donor 1 ate chocolate and drank black tea prior to creating the samples but did not rinse her mouth or drink water. Donor 2 drank coffee and ate crackers before donating saliva. He did not rinse his mouth or drink water before donating the sputum or licking any of the substrates.

The five samples (three blood, and two saliva) were also extracted using the EZ1 DNA Investigator kit and the BioRobot EZ1 Workstation (Qiagen, Hilden, Germany) following the method described earlier [19-21]. This method using the Qiagen instrument and the extraction kit is described in this manuscript as the 'traditional method'. Since this method of extraction is also robotic, is used in the authors' laboratories routinely, and has yielded reliable results, this method was chosen instead of using DNA IQ ${ }^{\mathrm{m} w}$ system for extraction. Quantification using the 7500 real time PCR $^{\circ}$ System (Applied Biosystems, Foster City, CA, USA) and the Quantifiler Human DNA Quantification kit (Life Technologies, Foster City, CA, USA) was 
performed following the recommended protocol [22]. An optimum amount of DNA was amplified with the reagents contained within the PowerPlex ${ }^{\oplus}$ 16HS System [5]. The generated profiles were used as reference profiles for comparison with the data generated from experiments performed and described later.

\section{Sensitivity of the instrument}

As described in Table 1 , the sensitivity and reproducibility study with the system was tested using various amounts of blood in order to determine the lowest volume needed to obtain complete profile. The following four dilutions were created from M1 and F1 blood samples; 1:1, 1:2, 1:4 and 1:8. The effective volumes of blood in each of these diluted samples are noted. A defined amount $(1.0 \mu \mathrm{L})$ of each of these diluted samples was spotted on a white sterilized porcelain spot plate. The samples were dried for 24 hours, swabbed with a sterile PurWraps ${ }^{\oplus}$ cotton swab which had been moistened with sterile distilled water. Each swab was dried for 24 hours. Each platform (one cartridge) contained four swabs in four separate channels. A sterile Pur-Wraps ${ }^{\oplus}$ swab containing $1.0 \mu \mathrm{L}$ of the neat liquid (undiluted) M1 blood sample was inserted into the fifth channel.

\begin{tabular}{|l|l|l|l|}
\hline \multirow{2}{*}{ Samples Run in Duplicate } & RapidHIT & \multicolumn{2}{|l|}{ Traditional Method } \\
\cline { 2 - 4 } & Volume of Blood $(\boldsymbol{\mu L})$ & Volume of Blood $(\boldsymbol{\mu L})$ & Average Quantity of DNA (ng) \\
\hline M1 and F1 & 1.0 & 1.0 & 1.2 \\
\hline M1 and F1 & 0.5 & 0.5 & 0.38 \\
\hline M1 and F1 & 0.25 & 0.25 & 0.28 \\
\hline M1 and F1 & 0.125 & 0.125 & 0.18 \\
\hline M1 and F1 & 0.0625 & 0.0625 & 0.15 \\
\hline
\end{tabular}

Table 1: Volumes and quantity of DNA from diluted blood samples for sensitivity study. Five different volumes of blood, ranging from 1.0-0.0625 $\mu \mathrm{L}$ from M1 and F1 samples, run in duplicates using the RapidHIT ${ }^{\text {sm }}$ System and the traditional method. The average quantity of DNA using the traditional method ranged from 1.2 to $0.15 \mathrm{ng}$.

For replication study, and as indicated in Table 1, the diluted blood samples were run twice using both M1 and F1 samples. Another set of swabs containing the same four diluted samples and one undiluted sample $(1.0 \mu \mathrm{L}$ blood) from M1 and F1 donors were extracted with the traditional method utilizing the EZ1 DNA Investigator kit (Table 1). This experiment was also repeated to determine the average quantity of DNA in each volume. The extracted DNA from these swabs was quantified as described earlier.
Table 2 denotes five different volumes of saliva, ranging from 50.0-10.0 $\mu \mathrm{L}$, obtained from Donor 1 and Donor 2. Each sample was deposited on a white sterilized porcelain spot plate and left to dry at room temperature for 24 hours prior to swabbing them with sterile, moistened Pur-Wraps swabs. As noted in the same table, the same volumes of saliva from both donors were extracted using the EZ1 DNA Investigator kit and the BioRobot EZ1 Workstation.

\begin{tabular}{|c|c|c|c|}
\hline \multirow[t]{2}{*}{ Samples Run in Duplicate } & \multirow{2}{*}{$\begin{array}{l}\text { RapidHIT }^{\mathrm{TM}} \\
\text { Volume of Saliva }(\mu \mathrm{L})\end{array}$} & \multicolumn{2}{|l|}{ Traditional Method } \\
\hline & & Volume of Saliva $(\mu \mathrm{L})$ & Average Quantity of DNA (ng) \\
\hline Donor 1 and Donor 2 & 50.0 & 50.0 & 977.0 \\
\hline Donor 1 and Donor 2 & 40.0 & 40.0 & 678.8 \\
\hline Donor 1 and Donor 2 & 30.0 & 30.0 & 393.3 \\
\hline Donor 1 and Donor 2 & 20.0 & 20.0 & 65.6 \\
\hline Donor 1 and Donor 2 & 10.0 & 10.0 & 33.3 \\
\hline
\end{tabular}

Table 2: Volumes and quantity of DNA from saliva samples for sensitivity study. Five different volumes of saliva, ranging from 50.0-10.0 $\mu \mathrm{L}$ from Donor 1 and Donor 2, run in duplicates using RapidHIT ${ }^{\mathrm{mox}}$ and the traditional method. The average quantity of DNA using the traditional method ranged from 977.0 to $33.3 \mathrm{ng}$.

\section{Simulated crime scene evidence samples commonly encountered in the UAE}

Experiments included various substrates, some of which contain potential inhibitors such as soil, and dyes [23-26]. All of the simulated crime scene substrates used in this study were cross-linked twice (one time for each side), using the Stratalinker 2400 UV Crosslinker at 9999 microjoules $\mathrm{x} 100$ prior to deposition of body fluids on them. Table 3 (blood samples) and Table 4 (saliva samples) list the substrates used in this study to mimic items of evidence commonly encountered in the indoor crime scenes in the UAE. The samples were prepared as described in these two tables. When necessary, the sterile swabs were moistened with distilled water prior to collection of the samples. 
Citation: Alshehhi A, Roy R (2015) Generating Rapid DNA Profiles from Crime Scene Samples Commonly Encountered in the United Arab

\begin{tabular}{|l|l|l|l|}
\hline Number & Crime Scene Substrates & Short Name & Collection Method \\
\hline 1 & Synthetic leather checkered pattern & Synthetic leather & $1.0 \mathrm{~cm}^{2}$ Cut \\
\hline 2 & Northern Red Oak branch covered with soil & Branch & Swabbed \\
\hline 3 & Pacon paper painted with Crayola washable water colors & Water paint & $1.0 \mathrm{~cm}^{2}$ Cut \\
\hline 4 & Denim jeans (100\% cotton) & Denim jeans & $1.0 \mathrm{~cm}^{2}$ Cut \\
\hline 5 & Great Value freezer bag (plastic) & Plastic bag & $1.0 \mathrm{~cm}^{2}$ Cut \\
\hline 6 & Card with Balspar Brand Premium Latex paint & Latex paint & Swabbed \\
\hline 7 & Natural Oak Parquet Gunstock Hardwood Flooring & Finished wood & Swabbed \\
\hline 8 & Ceramic floor tile & Tile & Swabbed \\
\hline 9 & Mexican Beach Pebble Stone covered with soil & Stone & Swabbed \\
\hline 10 & Tempered glass piece from a broken car window & Tempered glass & Swabbed \\
\hline 11 & Ash tree wood block (unfinished) & Unfinished wood & Swabbed \\
\hline 12 & Carpet Home Decorates Collections (2701 CARLSBAD) & Carpet fibers & 3 Fibers Cut \\
\hline 13 & Arabic Shimagh, Albassam (100\% white cotton) & Scarf & $1.0 \mathrm{~cm}^{2}$ Cut \\
\hline 14 & Pergo XP Ligoria Slate laminate flooring & Laminated flooring & Swabbed \\
\hline 15 & Kimberly-Clark® Kimwipe® & Kimwipe & $1.0 \mathrm{~cm}^{2}$ Cut \\
\hline
\end{tabular}

Table 3: Substrates used for deposition of blood and collection method. Fifteen substrates used for deposition of three different blood samples to mimic items of evidence commonly encountered in the indoor crime scenes in the UAE. Short names of the substrates are indicated in the column. The collection methods to gather this body fluid from these substrates are included.

\begin{tabular}{|c|c|c|c|}
\hline Number & Crime Scene Substrates (Saliva) & Short Name & Collection Method \\
\hline 1 & Stainless-steel spoon & Stainless-steel spoon & Licked/Swabbed \\
\hline 2 & Plastic fork & Plastic fork & Licked/Swabbed \\
\hline 3 & Plastic spoon & Plastic spoon & Licked/Swabbed \\
\hline 4 & Wrigley $5^{\circledR}$ Chewing Rain-gum & Minted chewing gum & Chewed $/ 3.0 \mathrm{~cm}^{2}$ Cut \\
\hline 5 & Self-adhesive stamp & Stamp & Licked $/ 1.0 \mathrm{~cm}^{2}$ Cut \\
\hline 6 & Chromite stone covered with soil & Stone & Spat on/Swabbed \\
\hline 7 & Starbucks ${ }^{\circledR}$ straw & Straw & Inserted in mouth $/ 1.0 \mathrm{~cm}^{2}$ Cut \\
\hline 8 & Adhesive part of self-seal envelope & Envelope & Licked/Swabbed \\
\hline 9 & Ice Mountain-bottle of water & Bottle & Inserted in mouth/Swabbed \\
\hline 10 & Glidden Trim and Door paint on index card & Oil paint & Spat on/Swabbed \\
\hline
\end{tabular}

Table 4: Substrates used for deposition of saliva and collection method. Undefined amounts of saliva from two donors deposited on 10 different substrates to mimic items of evidence commonly encountered in the indoor crime scenes in the UAE. Short names of the substrates are indicated in the column. The collection methods to gather this body fluid from these substrates are included.

Table 5 (blood samples) and Table 6 (saliva samples) indicate the experiments performed on each body fluid collected from the donors. Blood $(10.0 \mu \mathrm{L})$ from two deceased donors (F2 and M1) was deposited on each of these substrates and dried at room temperature for 24 hours, as described (Experiment 1, Table 5).
The saliva samples from the two donors (Experiment 1, Table 6) were deposited and then dried at room temperature $\left(24^{\circ} \mathrm{C}\right)$ for 24 hours. These substrates contained undefined amounts of saliva as they were prepared in an attempt to mimic crime scene samples. 
Citation: Alshehhi A, Roy R (2015) Generating Rapid DNA Profiles from Crime Scene Samples Commonly Encountered in the United Arab

\begin{tabular}{|c|c|c|c|c|}
\hline \multirow[t]{2}{*}{ Sample } & \multirow[t]{2}{*}{ Short Name } & \multicolumn{3}{|c|}{ Condition of The Experiment } \\
\hline & & Experiment $1(10.0 \mu \mathrm{L})$ & Experiment $2(10.0 \mu \mathrm{L})$ & Experiment $3(10.0 \mu \mathrm{L})$ \\
\hline F2 & Synthetic leather & $24^{\circ} \mathrm{C}$ & $48^{\circ}$ & $48^{\circ} \mathrm{C}+43 \%$ Humidity \\
\hline F2 & Branch & $24^{\circ} \mathrm{C}$ & $48^{\circ} \mathrm{C}$ & $48^{\circ} \mathrm{C}+43 \%$ Humidity \\
\hline F2 & Water paint & $24^{\circ} \mathrm{C}$ & $48^{\circ} \mathrm{C}$ & $48^{\circ} \mathrm{C}+43 \%$ Humidity \\
\hline F2 & Denim jeans & $24^{\circ} \mathrm{C}$ & $48^{\circ} \mathrm{C}$ & $48^{\circ} \mathrm{C}+43 \%$ Humidity \\
\hline F2 & Plastic bag & $24^{\circ} \mathrm{C}$ & $48^{\circ} \mathrm{C}$ & $48^{\circ} \mathrm{C}+43 \%$ Humidity \\
\hline M1 & Latex paint & $24^{\circ} \mathrm{C}$ & NT & NT \\
\hline M1 & Finished wood & $24^{\circ} \mathrm{C}$ & NT & NT \\
\hline M1 & Tile & $24^{\circ} \mathrm{C}$ & NT & NT \\
\hline M1 & Stone & $24^{\circ} \mathrm{C}$ & NT & NT \\
\hline M1 & Tempered glass & $24^{\circ} \mathrm{C}$ & NT & NT \\
\hline M1 & Unfinished wood & $24^{\circ} \mathrm{C}$ & $48^{\circ} \mathrm{C}$ & $48^{\circ} \mathrm{C}+43 \%$ Humidity \\
\hline M1 & Carpet fibers & $24^{\circ} \mathrm{C}$ & $48^{\circ} \mathrm{C}$ & $48^{\circ} \mathrm{C}+43 \%$ Humidity \\
\hline M1 & Scarf & $24^{\circ} \mathrm{C}$ & $48^{\circ} \mathrm{C}$ & $48^{\circ} \mathrm{C}+43 \%$ Humidity \\
\hline M1 & Laminated flooring & $24^{\circ} \mathrm{C}$ & $48^{\circ} \mathrm{C}$ & $48^{\circ} \mathrm{C}+43 \%$ Humidity \\
\hline M1 & Kimwipe & $24^{\circ} \mathrm{C}$ & $48^{\circ} \mathrm{C}$ & $48^{\circ} \mathrm{C}+43 \%$ Humidity \\
\hline
\end{tabular}

Table 5: Experiments used to test the effect of heat and humidity on $10.0 \mu \mathrm{L}$ of blood F2 and M1 blood samples (10.0 $\mu \mathrm{L})$ deposited on the listed substrates and subjected to three different conditions, which included room temperature, dry heat and heat and humidity factors.

\begin{tabular}{|c|c|c|c|c|c|}
\hline \multirow[t]{3}{*}{ Sample } & \multirow[t]{3}{*}{ Short Name } & \multicolumn{4}{|c|}{ Experimental Design } \\
\hline & & \multirow[t]{2}{*}{ Experiment 1} & \multirow[t]{2}{*}{ Experiment 2} & \multicolumn{2}{|c|}{ Experiment 3 (RapidHIT ${ }^{\mathrm{TM}}$ and Traditional Method) } \\
\hline & & & & Conditions & Duplicate Run \\
\hline Donor 1 & Stainless-steel spoon & $24^{\circ} \mathrm{C}$ & $48^{\circ} \mathrm{C}$ & $48^{\circ} \mathrm{C}+43 \%$ Humidity & Yes \\
\hline Donor 1 & Plastic fork & $24^{\circ} \mathrm{C}$ & $48^{\circ} \mathrm{C}$ & $48^{\circ} \mathrm{C}+43 \%$ Humidity & Yes \\
\hline Donor 1 & Plastic spoon & $24^{\circ} \mathrm{C}$ & $48^{\circ} \mathrm{C}$ & $48^{\circ} \mathrm{C}+43 \%$ Humidity & Yes \\
\hline Donor 1 & Gum & $24^{\circ} \mathrm{C}$ & $48^{\circ} \mathrm{C}$ & $48^{\circ} \mathrm{C}+43 \%$ Humidity & Yes \\
\hline Donor 1 & Stamp & $24^{\circ} \mathrm{C}$ & $48^{\circ} \mathrm{C}$ & $48^{\circ} \mathrm{C}+43 \%$ Humidity & Yes \\
\hline Donor 1 & Stone & $24^{\circ} \mathrm{C}$ & $N T^{*}$ & NT & NT \\
\hline Donor 1 & Straw & $24^{\circ} \mathrm{C}$ & NT & NT & NT \\
\hline Donor 1 & Envelope & $24^{\circ} \mathrm{C}$ & NT & NT & NT \\
\hline Donor 1 & Bottle & $24^{\circ} \mathrm{C}$ & NT & NT & NT \\
\hline Donor 1 & Oil paint & $24^{\circ} \mathrm{C}$ & NT & NT & NT \\
\hline Donor 2 & Stainless-steel spoon & NT & NT & $48^{\circ} \mathrm{C}+43 \%$ Humidity & Yes \\
\hline Donor 2 & Plastic fork & NT & NT & $48^{\circ} \mathrm{C}+43 \%$ Humidity & Yes \\
\hline Donor 2 & Plastic spoon & NT & NT & $48^{\circ} \mathrm{C}+43 \%$ Humidity & Yes \\
\hline Donor 2 & Gum & NT & NT & $48^{\circ} \mathrm{C}+43 \%$ Humidity & Yes \\
\hline
\end{tabular}


Citation: Alshehhi A, Roy R (2015) Generating Rapid DNA Profiles from Crime Scene Samples Commonly Encountered in the United Arab

\begin{tabular}{|l|l|l|l|l|l|}
\hline Donor 2 & Stamp & NT & NT & $48^{\circ} \mathrm{C}+43 \%$ Humidity & Yes \\
\hline
\end{tabular}

Table 6: Experiments used to test the effect of heat and humidity on saliva Undefined amounts of saliva from Donor 1 and Donor 2 deposited on different substrates and subjected to three different conditions which included room temperature, dry heat and heat and humidity factors. Samples were run in duplicate in Experiment 3, where indicated. Both the RapidHIT ${ }^{m}$ System and the traditional method were used in Experiment $3 .{ }^{*} \mathrm{NT}=$ Not Tested

The samples described as 'cut' were pieces of original substrates containing one body fluid. The size of each cut piece is noted in the tables. These cut pieces were introduced directly into the cartridges using the Accu-Chek Softclix lancet device (Roche, Pleasanton, CA, USA). The lancets were used to stabilize the samples at the bottom of the cartridges and also to minimize movement of the samples during extraction. The swabs were moistened and used to collect the body fluids. All cut samples and swabs were dried at room temperature for 24 hours prior to inserting each into one channel of the cartridge.

\section{Simulation of summer season conditions}

Data was obtained from the National Center of Meteorology and Seismology, Meteorological Department, Abu Dhabi, UAE [27]. The climate data was collected from two observation stations; Al Ain International Airport and Abu Dhabi International Airport. The weather report for the hottest four months in UAE, starting on May 1 st and ending on August 31st indicated that the highest temperature was at $48.5^{\circ} \mathrm{C}$. The relative humidity was also recorded for those days.
Based on that data from two Summer Seasons the average percent of humidity was calculated to be $42.66 \%$. Therefore, samples containing blood and saliva were left at $48^{\circ} \mathrm{C}$ temperature for 24 hours. This condition was referred to as 'dry heat' in this manuscript.

Table 5 lists the blood samples that were exposed to either dry heat (Experiment 2) or hot and humid conditions of $48^{\circ} \mathrm{C}$ and $43 \%$ relative humidity for 24 hours (Experiment 3). Each of these samples contained $10.0 \mu \mathrm{L}$ of blood from either M1 or F2 individuals. Table 6 includes saliva samples that were exposed to the same conditions as described above. An acu-Rite 00325 Home Comfort Monitor hygrometer was used to measure the humidity percentage throughout the incubation process. The temperature and humidity remained constant throughout the duration of this research.

Table 7 lists the samples containing $3.0 \mu \mathrm{L}$ of blood from the three deceased donors, F1, F2 and M1. Where noted, each sample was subjected to heat and humidity conditions. For reproducibility study, 10 of these samples were run in duplicates.

\begin{tabular}{|c|c|c|c|c|}
\hline \multicolumn{5}{|c|}{ Experiment $4(3.0 \mu \mathrm{L}$ Blood $)$} \\
\hline \multirow[t]{2}{*}{ Sample } & \multirow[t]{2}{*}{ Substrates } & \multirow[t]{2}{*}{ Collection Method } & \multirow[t]{2}{*}{ Conditions } & RapidHIT ${ }^{\mathrm{TM}}$ and Traditional Method \\
\hline & & & & Duplicate Run \\
\hline F2 & Synthetic leather & $1.0 \mathrm{~cm}^{2}$ Cut & $48^{\circ} \mathrm{C}+43 \%$ Humidity & Yes \\
\hline F2 & Denim jeans & $1.0 \mathrm{~cm}^{2}$ Cut & $48^{\circ} \mathrm{C}+43 \%$ Humidity & Yes \\
\hline F2 & Branch & Swabbed & $48^{\circ} \mathrm{C}+43 \%$ Humidity & NT \\
\hline F2 & Water paint & $1.0 \mathrm{~cm}^{2}$ Cut & $48^{\circ} \mathrm{C}+43 \%$ Humidity & NT \\
\hline F2 & Plastic bag & $1.0 \mathrm{~cm}^{2}$ Cut & $48^{\circ} \mathrm{C}+43 \%$ Humidity & NT \\
\hline M1 & Unfinished wood & Swabbed & $48^{\circ} \mathrm{C}+43 \%$ Humidity & Yes \\
\hline M1 & Carpet fibers & 3 Fibers Cut & $48^{\circ} \mathrm{C}+43 \%$ Humidity & Yes \\
\hline M1 & Scarf & $1.0 \mathrm{~cm}^{2}$ Cut & $48^{\circ} \mathrm{C}+43 \%$ Humidity & Yes \\
\hline M1 & Laminated flooring & Swabbed & $48^{\circ} \mathrm{C}+43 \%$ Humidity & NT \\
\hline M1 & Kimwipe & $1.0 \mathrm{~cm}^{2}$ Cut & $48^{\circ} \mathrm{C}+43 \%$ Humidity & NT \\
\hline F1 & Synthetic leather & $1.0 \mathrm{~cm}^{2}$ Cut & $48^{\circ} \mathrm{C}+43 \%$ Humidity & Yes \\
\hline F1 & Denim jeans & $1.0 \mathrm{~cm}^{2}$ Cut & $48^{\circ} \mathrm{C}+43 \%$ Humidity & Yes \\
\hline F1 & Unfinished wood & Swabbed & $48^{\circ} \mathrm{C}+43 \%$ Humidity & Yes \\
\hline F1 & Carpet fibers & 3 Fibers Cut & $48^{\circ} \mathrm{C}+43 \%$ Humidity & Yes \\
\hline
\end{tabular}




\begin{tabular}{|l|l|l|l|l|}
\hline F1 & Scarf & $1.0 \mathrm{~cm}^{2} \mathrm{Cut}$ & $48^{\circ} \mathrm{C}+43 \%$ Humidity & Yes
\end{tabular}

Table 7: Experiment 4 used to test the effect of heat and humidity on $3.0 \mu \mathrm{L}$ of blood F2, F1 and M1 blood samples (3.0 $\mu \mathrm{L})$ deposited on various substrates and subjected to heat and humidity conditions. As indicated, ten of these samples were run in duplicate for reproducibility study. Both the RapidHIT ${ }^{\mathrm{mex}}$ System and the traditional method were used in this experiment.

\section{Traditional STR typing protocols}

Each sample subjected to analysis by the RapidHIT ${ }^{\text {in }}$ System was also analyzed by standard method used in the authors' research laboratories using procedures described previously [19-21]. Briefly, all samples including the reference samples (blood and saliva) were extracted using the EZ1 DNA Investigator kit and the BioRobot EZ1 Workstation. After quantification of the extracted DNA,

0.5 ng DNA was amplified using PowerPlex ${ }^{\circ} 16$ HS STR System. However, instead of the recommended reaction volume of $25 \mu \mathrm{L}$, these samples were amplified in a reduced reaction volume of $12.5 \mu \mathrm{L}$. DNA was injected for fragment analysis on the 3130xl Genetic Analyzer from Applied Biosystems ${ }^{\oplus}$, following the manufacturer's recommendation [5].

\section{Analysis of data}

Data analysis was completed using GeneMarker ${ }^{\oplus}$ HID software version 2.7.1 from SoftGenetics ${ }^{\oplus}$. The RStudio package (Version 0.98 .1048 ) was utilized to analyze the peak height ratios that were called by the GeneMarker ${ }^{\bullet}$ HID software [28]. Analytical threshold (AT) was set by default parameters in the analysis software on the RapidHIT $^{\text {tx }}$ System based on the interpretation used previously [17]. Depending on the baseline peaks at each locus, the AT was set at five times the standard deviation of the average baseline RFU values. For homozygote loci, the stochastic threshold was set at two times the AT. Also, the software considers heterozygote balance (PHR) of less than $50 \%$ as unreliable, while peaks that are between $50 \%$ and $70 \%$ were flagged for further analysis. The default settings of AT and PHR were used in this study. These parameters can be changed according to each laboratory's protocol and internal validation requirements.

\section{Results and Discussion}

The recently introduced 'Run Other Sample' instrument protocol was used in this research for processing and analyzing the blood and saliva samples. Although this instrument was originally developed to obtain STR profiles from buccal swabs only, this protocol made it possible to analyze both blood and saliva samples.

\section{Sensitivity study}

Tables 1 and 2 include the experiments conducted to determine the sensitivity of the instrument when using blood and saliva samples. Table 1 lists the average quantities of DNA in the undiluted $(1.0 \mu \mathrm{L})$ of blood and the four diluted samples $(0.5,0.25,0.125,0.0625 \mu \mathrm{L})$. The average quantities of DNA listed were derived from the amounts of DNA obtained from the blood samples (M1 and F1) and their replicate runs.

Figure 1 demonstrates the average percentages of called alleles from the five different volumes of blood listed in Table 1. This figure shows a correlation between the volumes of blood and the percentages of called alleles. In the first run with the F1 sample, the PHR at the FGA locus was $46 \%$. However, as shown in Table 8 , in the duplicate run with the F1 sample as well as both runs of the M1 sample, complete profiles were obtained from $0.25 \mu \mathrm{L}$ of blood. Partial profiles were obtained when the volumes of blood were less than $0.25 \mu \mathrm{L}$. The average percentages of profile recovery from $0.25,0.125$ and $0.0625 \mu \mathrm{L}$ were $99 \pm 0.9,90 \pm 3.2$ and $88 \pm 3.3$, respectively. This study indicated that $0.28 \mathrm{ng}$ of DNA ( $0.25 \mu \mathrm{L}$ of blood) was the minimum amount needed to obtain complete DNA profiles from the blood samples obtained from the two deceased donors. Figure 2 shows a complete autosomal STR profile obtained from $0.25 \mu \mathrm{L}$ of blood using the RapidHIT ${ }^{\mathrm{sm}}$ System.

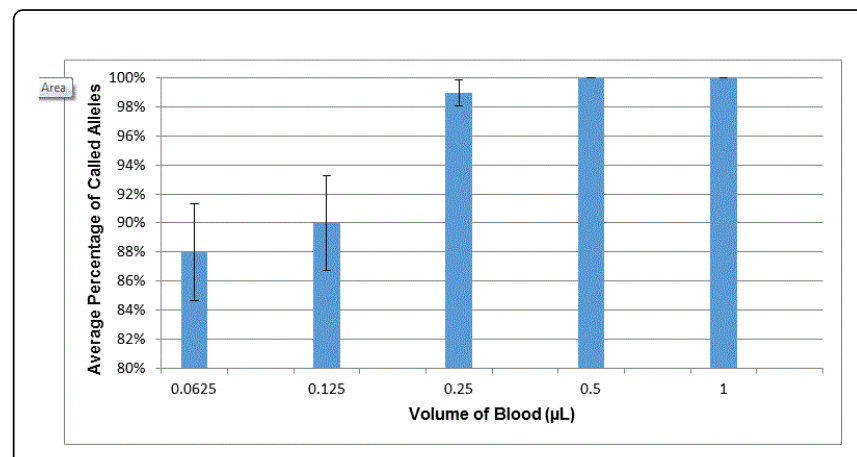

Figure 1: The average percentages of called alleles from five different volumes of blood showing a correlation between the volumes of blood and the percentages of called alleles

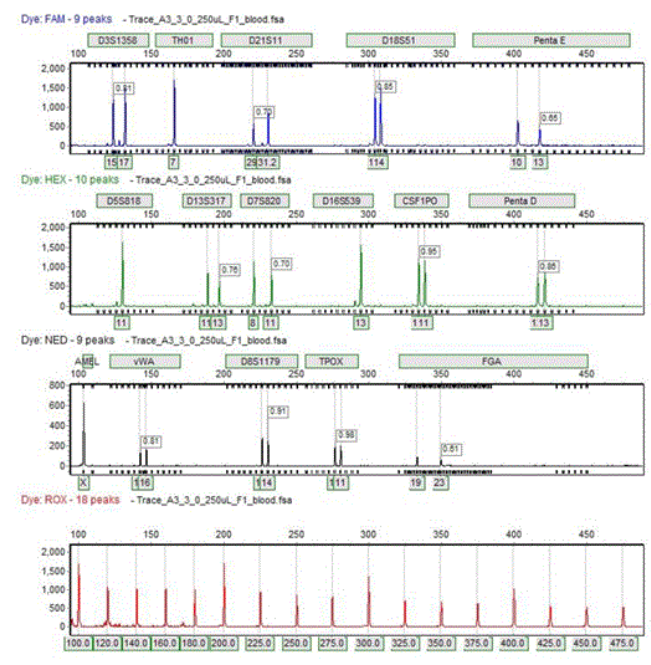

Figure 2: A complete autosomal STR profile obtained from $0.25 \mu \mathrm{L}$ of blood using the RapidHIT ${ }^{\mathrm{mu}}$ System 


\begin{tabular}{|l|l|l|l|l|}
\hline Sample & Volume of Blood $(\boldsymbol{\mu L})$ & Run & Allele Dropout & Loci with PHR Below 50\% \\
\hline F1 & 0.0625 & Run1 & $\begin{array}{l}\text { D3S1358 }(1 / 2)^{* *}, \text { TPOX (1/2) and } \\
\text { FGA }(2 / 2)^{* * *}\end{array}$ & VWA (18\%) and D7S820 (36\%) \\
\hline M1 & 0.0625 & Run1 & No dropout & D5S818 (37\%) and D16S539 (47\%) \\
\hline F1 & 0.125 & Run1 & D16S539 (1/2) and FGA (2/2) & CSF1PO (25\%) \\
\hline M1 & 0.125 & Run1 & No dropout & CSF1PO (34\%) \\
\hline F1 & 0.25 & Run1 & No dropout & FGA (46\%) \\
\hline M1 & 0.25 & Run1 & No dropout & No dropout \\
\hline F1 & 0.0625 & Run2 & No dropout & D21S11 (22\%), D18S51 (28\%) and CSF1PO (40\%) \\
\hline M1 & 0.0625 & Run2 & Amelogenin (X allele) & TH01 (38\%), D18S51 (41\%) and Penta D (30\%) \\
\hline F1 & 0.125 & Run2 & No dropout & $\begin{array}{l}\text { Penta E (43\%), D13S317 (46\%), D7S820 (47\%) and TPOX } \\
(46 \%)\end{array}$ \\
\hline M1 & 0.125 & Run2 & No dropout & D3S1358 (38\%), D21S11 (48\%) and Penta D (47\%) \\
\hline F1 & 0.25 & Run2 & No dropout & No dropout \\
\hline M1 & 0.25 & Run2 & No dropout & No dropout \\
\hline
\end{tabular}

Table 8: Allele dropouts from the sensitivity study using blood. Allele dropouts noted in profiles from the sensitivity study using various volumes of blood (Table 1). Duplicate experiment with the same samples is noted as Run 2. Dropout alleles include peaks with PHRs less than 50\% in heterozygous loci, and the peaks that were not called by the software because they had RFU values lower than the analytical threshold. ${ }^{\star}$ One allele considered to be dropped out in the percentage of called alleles calculation, ${ }^{* *}$ One allele dropout in a heterozygous locus, ${ }^{* *}$ Both alleles dropout in a heterozygous locus

Table 8 show allele dropouts in profiles obtained from the diluted blood samples. Duplicate experiment with the same samples is noted as Run 2. Dropout alleles include peaks with PHRs less than $50 \%$ in heterozygous loci, and the peaks that were not called by the software because they had RFU values lower than the AT, which was set by default parameters. Tables 8 demonstrate that allele dropouts were observed in loci with larger amplicon sizes, such as Penta D, Penta E, and FGA, as well as in loci with smaller amplicon sizes, such as D18S51, vWA, TH01, and TPOX. These results indicate that increased allelic dropout with less than $0.25 \mu \mathrm{L}$ of blood is possibly due to stochastic sampling effects or preferential binding, which is commonly experienced with low amounts of DNA [29]. In addition, drop-in peaks were not observed in the profiles generated from diluted and undiluted blood samples using the RapidHIT ${ }^{\mathrm{ix}}$ System. All profiles were concordant with the reference profiles obtained using the traditional STR typing.

Table 2 shows average quantities of DNA from saliva samples ranging in volumes from 50.0 to $10.0 \mu \mathrm{L}$, processed using the RapidHIT $^{\text {mo }}$ System and the traditional method. Saliva samples were obtained from two living donors, and all samples were run in duplicates. As shown in Table 2, the average quantity of DNA in these five different volumes of samples ranged from 977.0 to $33.3 \mathrm{ng}$.

Complete profiles were generated from all volumes of saliva samples using both the RapidHIT ${ }^{\mathrm{mm}}$ System and the traditional method (data not shown). The data was concordant between the two STR typing methods. These results indicate that complete STR profiles can be generated using the automated system from saliva samples that contain at least $33 \mathrm{ng}$ of DNA.

\section{Simulated crime scene evidence samples commonly encountered in the UAE}

DNA profiles were successfully generated from all blood samples that were dried at room temperature described in Experiment 1, Table 5 (data not shown). In addition, each saliva sample listed in Experiment 1, Table 6 and dried at room temperature, yielded complete STR profiles using the RapidHIT ${ }^{\text {tx }}$ System (data not shown). These STR profiles were concordant with the reference profiles generated using the traditional method.

\section{Potential inhibitors and the robustness of the system}

To determine the robustness of the system, some of the substrates listed in Table 3 contained potential inhibitors [23-26]. Paints and colors used to coat papers, soil on wood and stones, and denim jeans used in these experiments did not cause inhibition when DNA profiles were generated from blood by automated system or by the traditional method. Although blood deposited on denim jeans initially generated a partial profile with the automated system, when this experiment was repeated a complete profile was obtained from the bloodstain on the jeans. All of the other samples containing potential inhibitors yielded complete profiles. Similarly, complete profiles were also obtained from the saliva samples collected from the two donors and listed in Table 4 (data not shown). As mentioned earlier, both donors consumed food and drinks such as chocolate, crackers, black tea, and coffee, immediately before donating sputum. This experiment shows that the system is robust and potential inhibitors commonly used in the UAE did not interfere with the amplification of the DNA. 


\section{Simulation of Summer Season conditions}

When the evidence samples were created with $10.0 \mu \mathrm{L}$ of F2 and M1 blood samples which were then exposed to dry heat (Experiment 2, Table 5) complete and concordant profiles were obtained. PHR values in these profiles ranged from $67-100 \%$.

Experiment 3, Table 5, also included $10.0 \mu \mathrm{L}$ of blood from F2 and M1 samples, deposited on the same 10 substrates as used in Experiment 2. These were exposed to heat and humidity factors as noted.

In Experiment 4, Table 7, $3.0 \mu \mathrm{L}$ of blood from F2, F1 and M1 samples were deposited on the same 10 substrates and exposed to the same conditions of heat and humidity. All of the samples in these two experiments were incubated at $48^{\circ} \mathrm{C}$ heat and $43 \%$ relative humidity for 24 hours to simulate UAE Summer Season conditions encountered in indoor crime scenes in this country.

Figure 3 is a boxplot comparing the average PHR of profiles generated from $10.0 \mu \mathrm{L}$ of blood, from $\mathrm{F} 2$ and $\mathrm{M} 1$ samples and deposited on nine substrates (Experiment 3, Table 5). The results of these samples are shown as the blue bars. As indicated by the red bars, this figure also compares the PHR of profiles generated from $3 \mu \mathrm{L}$ of blood using the same two samples and deposited on the same nine substrates (Experiment 4, Table 7).

As shown in Figure 3, most PHRs were above 70\% for both volumes of blood. However, 3.0 $\mu \mathrm{L}$ of blood deposited on denim jeans and synthetic leather yielded lower PHRs. Overall, all peak height ratios in profiles generated from these substrates were above $50 \%$. During the first run $10.0 \mu \mathrm{L}$ of blood from F2 sample, deposited on the plastic bag, did not show any profile due to injection failure (data not shown).

However, when this experiment was repeated using the same amount of blood from the same F2 sample and deposited in a different area on the same plastic bag, using the same instrument, the sample yielded complete profile. Figure 3 does not include the data from the plastic bag.

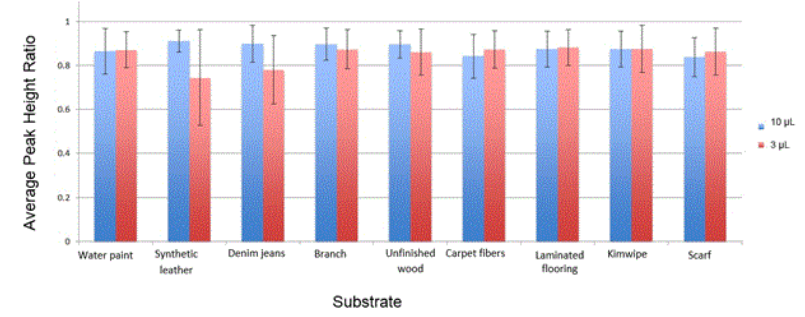

Figure 3: Boxplot comparing the average PHR of profiles generated from $10.0 \mu \mathrm{L}$ and $3.0 \mu \mathrm{L}$ of blood from $\mathrm{F} 2$ and M1 samples deposited on nine substrates

These conditions can vary for outdoor crime scenes due to exposure to sun and dust. The current study included only indoor conditions under which evidence was exposed for 24 hours in order to compare the results between the different experiments.

Although not tested, the time and environmental factors may further affect the results. Time-based experiments and environmental factors would be taken into consideration for future studies.
Figure 4 shows a complete profile obtained from $3.0 \mu \mathrm{L}$ of F2 blood sample deposited on denim jeans and exposed to $48^{\circ} \mathrm{C}$ heat and $43 \%$ relative humidity for 24 hours. This profile was obtained using the RapidHIT ${ }^{\text {Tw }}$ System.

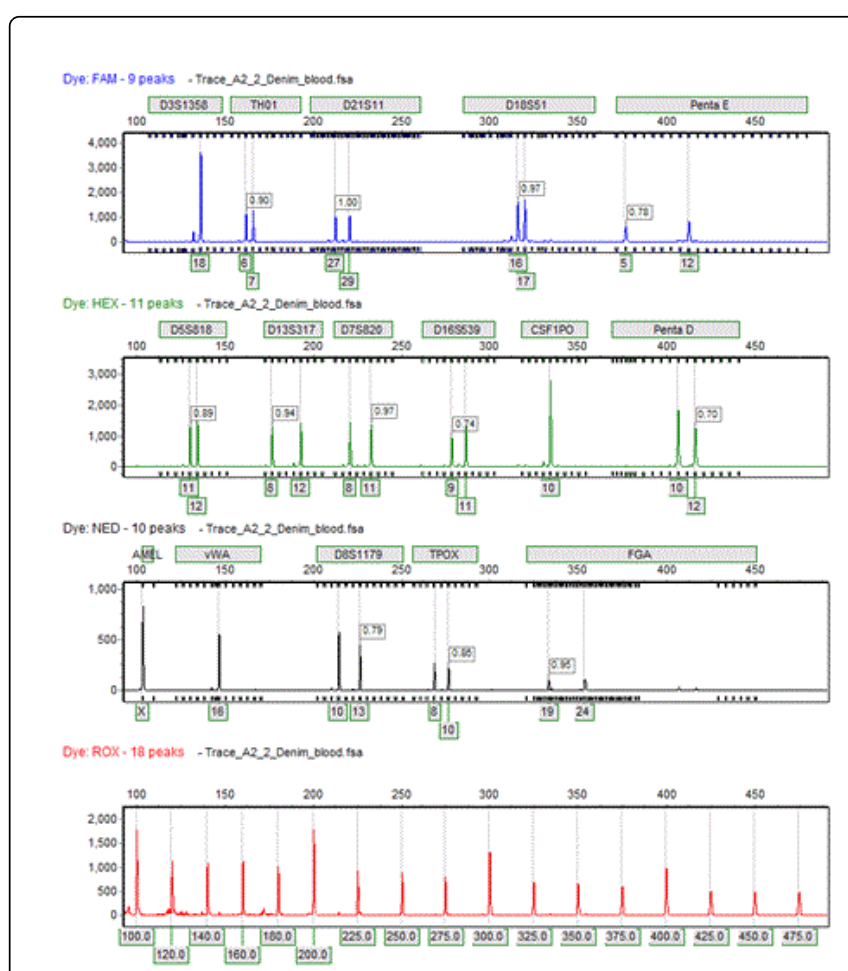

Figure 4: A complete autosomal STR profile obtained from $3.0 \mu \mathrm{L}$ of F2 blood sample deposited on denim jeans and exposed to $48^{\circ} \mathrm{C}$ heat and $43 \%$ relative humidity for 24 hours. This profile was obtained using the PowerPlex ${ }^{\star} 16$ HS STR System cartridge in the RapidHIT ${ }^{\text {mat }}$ System

Figure 5 compares heterozygote balance in profiles generated using 3.0 $\mu \mathrm{L}$ of blood from F1, F2 and M1 samples. The blood samples were deposited on the substrates listed in Table 7.

These bloodstains were incubated at $48^{\circ} \mathrm{C}$ heat and $43 \%$ humidity for 24 hours. Most PHR values were at or above $60 \%$. Wider range of PHR values were observed in the two largest loci in the multiplex (Penta E, and FGA) from blood deposited on the synthetic leather, and in D16S539 locus from bloodstain on the denim jeans.

This suggests that the synthetic leather and the denim jeans, when subjected to heat and humidity factors used in this study possibly have inhibitory effects on the blood samples. Since Penta E and FGA loci are the largest amplicons they tend to drop out more frequently in samples that are degraded or inhibited [30].

However, $10.0 \mu \mathrm{L}$ of the blood samples deposited on the synthetic leather yielded PHR values ranging from $83-95 \%$ when considering all of the loci. Similarly, the PHR values for denim jeans were also higher and ranged from $74-97 \%$ (data not shown). As expected, larger volumes of blood yielded more balanced alleles. 


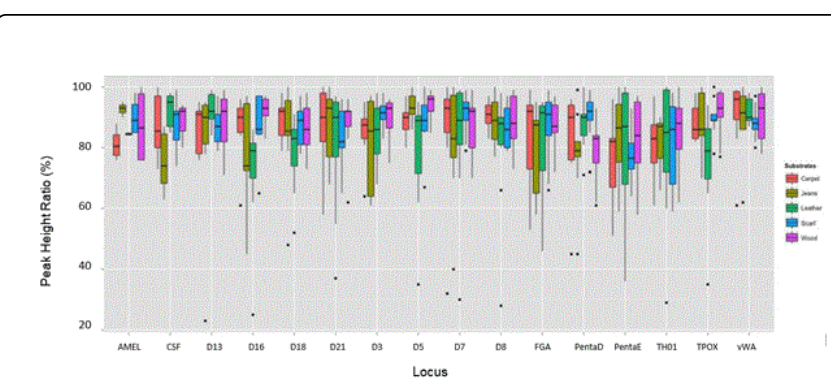

Figure 5: Comparison of heterozygote balance in profiles generated using 3.0 $\mu \mathrm{L}$ of blood from F1, F2 and M1 samples. The blood samples were deposited on five substrates and run in duplicates. These bloodstains were incubated at $48^{\circ} \mathrm{C}$ heat and $43 \%$ humidity for 24 hours

When saliva samples were subjected dry heat only (Experiment 2, Table 6) DNA profiles were successfully generated. The PHR values for all of these samples ranged from $61-100 \%$. Similarly, complete and concordant DNA profiles generated from the saliva samples which were exposed to heat and humidity conditions (Experiment 3, Table 6) yielded the same range of PHR values as the samples which were exposed to dry heat only (Experiment 2, Table 6). In Experiment 4, Table 7, no statistically significant difference of PHR values were noted within duplicate runs of the same samples $(p=0.1394)$ and between the saliva samples collected from two donors deposited on the same substrates $(\mathrm{p}=0.1007)$. Concordance between these STR profiles was established when compared with profiles generated using the traditional method.

\section{Concordance study}

All data generated with the RapidHIT ${ }^{\text {ma }}$ System were concordant with the data generated with the traditional STR typing method, indicating that this automated system is compatible and reliable. Figure 6 is a boxplot comparing the heterozygote balance of the STR profiles generated with traditional typing method and the RapidHIT ${ }^{\mathrm{rm}}$ instrument. In each method, 3.0 $\mu \mathrm{L}$ of blood from M1, F1 and F2 samples were deposited on these five substrates; carpet, jeans, leather, scarf, and wood.

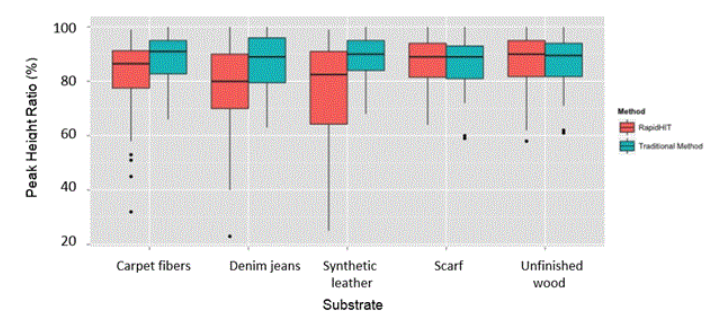

Figure 6: Boxplot comparing the heterozygote balance of the STR profiles generated with traditional typing method and the RapidHIT $^{\text {mo }}$ instrument. In each method, $3.0 \mu \mathrm{L}$ of blood from M1, F1 and F2 samples were deposited on five substrates. These were then subjected to $48^{\circ} \mathrm{C}$ heat and $43 \%$ relative humidity for 24 hours

These were then subjected to $48^{\circ} \mathrm{C}$ heat and $43 \%$ relative humidity for 24 hours. As observed, PHR values of the profiles generated using the traditional method were above $80 \%$ and those generated using the automated instrument were above $65 \%$. Also, more outliers and wider range of PHRs were noted in profiles generated using the RapidHIT ${ }^{\mathrm{Tm}}$ System. The difference in the peak height ratios generated by the two methods was statistically significant $(\mathrm{p}=1.548 \mathrm{e}-11)$.

It should be noted that each method had its advantages and shortcomings. While the traditional method yielded better quality profiles, as indicated in Figures 6 and 7, this procedure also involved many more steps and was more time-consuming compared to the RapidHIT ${ }^{\text {Tx }}$ System.

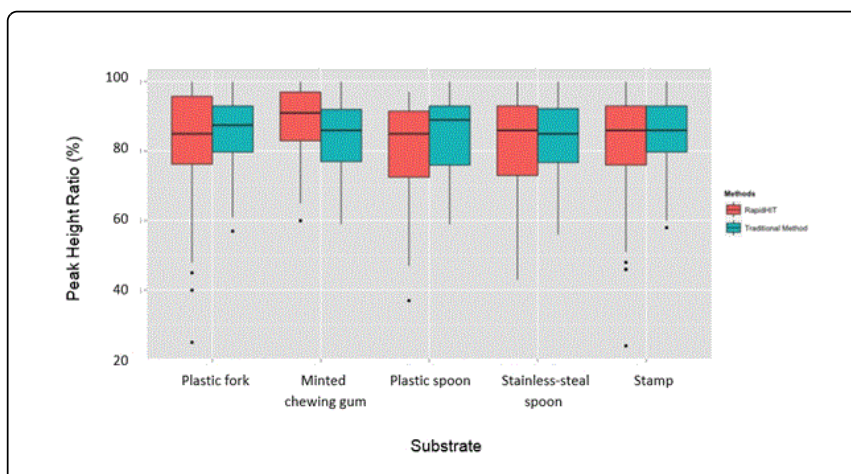

Figure 7: Boxplot comparing the heterozygote balance of the STR profiles generated with traditional typing method and the RapidHIT $^{\text {mis }}$ instrument. In each method, saliva from Donor 1 and Donor 2 were deposited on five substrates. These were then subjected to $48^{\circ} \mathrm{C}$ heat and $43 \%$ relative humidity for 24 hours

\section{Contamination between channels or samples}

Forensic crime laboratories routinely separate the pre-amplification and post-amplification areas to avoid any issues with contamination. No contamination was observed in the positive control channel, in the ladder channel or in the five evidence channels in each cartridge of the RapidHIT $^{\mathrm{mm}}$ System. Examination of the profiles from all runs and all cartridges indicated that only single source profiles were generated from each channel, and each profile was consistent with the donor's known profile.

However, two negative channels showed two different and complete single source STR profiles. These profiles could not be traced back to any of the reference samples, to the two researchers who authored this study, or to anyone in the IntegenX employees' database. The alleles in these two profiles were much less intense and did not have high RFU values (data not shown). Similar phenomenon has been reported before using this automated system and also for standard STR genotyping performed in the laboratory [17,31-33].

\section{Conclusion}

Results of the dilution study in duplicate indicated that approximately $0.28 \mathrm{ng}$ of DNA $(0.25 \mu \mathrm{L}$ of blood $)$ and $33.3 \mathrm{ng}$ of DNA $(10.0 \mu \mathrm{L}$ of saliva) were needed to generate complete autosomal STR profiles. With the automated system, allelic dropouts were observed only in cases where the volumes of the body fluids and the quantity of DNA were less than noted above.

Some of the substrates tested in this study contained potential inhibitors such as dyes in the denim jeans and paints, as well as 
substrates which contained soil. These substrates had no effect on the typing results.

The heat and humidity conditions used in this study did not adversely affect the RapidHIT ${ }^{\mathrm{m}}$ System's ability to yield complete and concordant profiles. The results from these experiments indicate that this instrument can be used successfully in investigating crimes committed indoors and in countries whether the weather conditions are similar to that found in the UAE.

Except for the two negative control samples that yielded complete profiles, contamination was not observed between channels in the same cartridge and between consecutive runs on the same instrument.

Data generated using the traditional method for extraction, quantification and amplification using the PowerPlex 16 HS STR System was in complete concordance with the data generated with RapidHIT $^{\mathrm{m}}$ System using the "Run Other Samples" instrument protocol. Comparison of the quality of the generated profiles indicated that the results were concordant and reliable within and between samples and with the profiles generated by traditional method.

The advantages of having all of the steps of DNA analysis in one fully integrated instrument, such as the RapidHIT ${ }^{\mathrm{m}}{ }^{\mathrm{m}}$ Human DNA Identification System, are several. The device is user-friendly, and portable. The instrument can be used by non-scientists at the crime scene after proper training, or in a forensic laboratory by the DNA analysts, immediately after the samples are collected. The entire process takes approximately five minutes of hands-on time. Additionally, DNA STR profiles can be generated within approximately 90 minutes.

\section{Acknowledgement}

The authors acknowledge receiving technical assistance and advice from Dr. Stefanie Gangano, Director of Technical Account Management at IntegenX and many other individuals from the company, helpful comments received from research committee members Ralph Ristenbatt, faculty at The Pennsylvania State University, and Mohammed Almazrooei, Lieutenant at the Forensic Department in the Abu Dhabi Police Biology Laboratory. The authors are particularly grateful to the Police Division in the Embassy of UAE in Washington, DC for their valuable contribution to this project.

Generous guidance was provided by Dr. Teresa Snyder-Leiby, (Product Manager at SoftGenetics LLC,), Mark Guilliano and Mary Jones Dukes (Qiagen) and Dawn Waltman (Thermo Fisher Scientific, Inc.).

\section{Ethical Disclosures}

The authors have no financial conflict or disclosures to state regarding this research. All samples were collected as approved by the Institutional Review Board (IRB) and Institutional Biosafety Committee (IBC) of The Pennsylvania State University, University Park, Pennsylvania. All samples were anonymized.

\section{References}

1. Moretti TR, Baumstark AL, Defenbaugh DA, Keys KM, Budowle B, et al. (2001) Validation of STR typing by capillary electrophoresis. J Forensic Sci 46: 661-676.

2. Moretti TR, Baumstark AL, Defenbaugh DA, Keys KM, Budowle B, et al. (2001) Validation of short tandem repeats (STRs) for forensic usage: performance testing of fluorescent multiplex STR systems and analysis of authentic and simulated forensic samples. J Forensic Sci 46: 647-660.

3. Krenke BE, Tereba A, Anderson SJ, Buel E, Culhane S, et al. (2002) Validation of a 16-locus fluorescent multiplex system. J Forensic Sci 47: 773-785.

4. Collins PJ, Hennessy LK, Leibelt CS, Roby RK, Reeder DJ, et al. (2004) Developmental validation of a single-tube amplification of the 13 CODIS STR loci, D2S1338, D19S433, and amelogenin: the AmpFISTR Identifiler PCR amplification kit. J. Forensic Sci 49: 1265-1277.

5. Ensenberger MG, Thompson J, Hill B, Homick K, Kearney K, et al. (2010) Developmental validation of the PowerPlex 16 HS System: an improved 16-locus fluorescent STR multiplex. Forensic Sci. Int. Genet 4: 257-264.

6. Wang D, Chang C, Lagacé R, Calandro L, Hennessy L (2012) Developmental validation of the AmpFISTR Identifiler Plus PCR Amplification Kit: an established multiplex assay with improved performance. J Forensic Sci 57: 453-465.

7. Ensenberger M, Hill C, McLaren R, Sprecher C, Storts D (2014) Developmental validation of the PowerPlex 21 System. Forensic Sci. Int. Genet 9: 169-178.

8. Wang D, Chang C, Oldroyd N, Hennessy L (2009) Direct amplification of STRs from blood or buccal cell samples. Forensic Sci Int 2: 113-114.

9. Wang DY, Chang CW, Lagacé RE, Oldroyd NJ, Hennessy LK (2011) Development and Validation of the AmpFeSTR ${ }^{\circledast}$ Identifiler $^{\circledR}$ Direct PCR Amplification Kit: A Multiplex Assay for the Direct Amplification of Single-Source Samples. J Forensic Sci 56: 835-845.

10. Myers B, King J, Budowle B (2012) Evaluation and comparative analysis of direct amplification of STRs using PowerPlex ${ }^{\oplus} 18 \mathrm{D}$ and Identifiler Direct systems. Forensic Sci Int. Genet 6: 640-645.

11. Hall D, Roy R (2014) An evaluation of direct PCR amplification. Croat Med J 55: 655-661.

12. Altshuler H, Roy R Evaluation of direct PCR amplification using various swabs and washing Reagents. J Forensic Sci (In press).

13. Jaffredo F, Freund F, Godichaud S, Gaboyard M, Moisan J (2011) Complete automated DNA procedure to facilitate DNA database collection. Forensic Sci Int: Genet Suppl Ser 3: e228-e229.

14. Gangano S, Elliott SK, Anoruo K, Gass J, Buscaino J, et al. (2013) DNA investigative lead development from blood and saliva samples in less than two hours using the RapidHIT ${ }^{\mathrm{Tm}}$ Human DNA Identification System. Forensic Sci Int: Genet Suppl Ser 4: e43-e44.

15. Hennessy LK, Franklin H, Li Y, Buscaino J, Chear K, et al. (2013) Developmental validation studies on the RapidHIT ${ }^{\mathrm{Tm}}$ Human DNA Identification System. Forensic Sci Int: Genet Suppl Ser 4: e7-e8

16. Tan E, Turingan RS, Hogan C, Vasantgadkar S, Palombo L, et al. (2013) Fully integrated, fully automated generation of short tandem repeat profiles. Investig Genet 4: 16.

17. LaRue BL, Moore A, King JL, Marshall PL, Budowle B (2014) An evaluation of the RapidHIT ${ }^{\text {Tx }}$ System for reliably genotyping reference samples. Forensic Sci Int Genet 13: 104-111.

18. Holland MM, Wendt F (2015) Evaluation of the RapidHIT ${ }^{\mathrm{ma}}$ 200, an automated human identification system for STR analysis of single source samples. Forensic Sci Int Genet 14: 76-85.

19. Ho TT, Roy R (2011) Generating DNA profiles from immunochromatographic cards using LCN methodology. For. Sci Int. Genet 5: 210-215.

20. Klein R, Neumann C, Roy R. (2015) Detecting Insertion Deletion Polymorphism Using DIPplex kit. Forensic Sci Int. Genet 16: 29-37.

21. DNA investigator handbook. Hilden, Germany: Qiagen (2010).

22. Applied Biosystems Quantifiler user's manual. Foster City, CA. (2005).

23. Opel K, Chung KD, McCord B (2010) A Study of PCR Inhibition Mechanisms Using Real Time PCR. J Forensic Sci 55: 25-33.

24. Demeke T, Jenkins GR (2010) Influence of DNA extraction methods, PCR inhibitors and quantification methods on real-time PCR assay of biotechnology-derived traits. Anal Bioanal Chem 396: 1977-1990. 
Citation: Alshehhi A, Roy R (2015) Generating Rapid DNA Profiles from Crime Scene Samples Commonly Encountered in the United Arab Emirates. J Forensic Res 6: 1000296. doi:10.4172/2157-7145.1000296

Page 12 of 12

25. Funes-Huacca M, Opel K, Thompson R, McCord B (2011) A comparison of the effects of PCR inhibition in quantitative PCR and forensic STR analysis. Electrophoresis 32: 1084-1089.

26. Martellossi C, Taylor EJ, Lee D, Graziosi G, Donini P (2005) DNA extraction and analysis from processed coffee beans. J. Agric. Food Chem 53: 8432-8436.

27. National Center of Meteorology \& Seismology, Meterological department, PO Box 4815 Abu Dhabi, UAE.

28. Studio R (2012) "RStudio: Integrated development environment for R" (Version 0.98.1048) [Computer software]. Boston, MA.

29. Timken MD, Klein SB, Buoncristiani MR (2014) Stochastic sampling effects in STR typing: Implications for analysis and interpretation. Forensic Sci Int Genet 11: 195-204.
30. Ge J, Eisenberg A, Budowle B (2012) Developing criteria and data to determine best options for expanding the core CODIS loci. Investigative Genetics 3: 2041-2223.

31. Schmidt, T., Hummel S, Herrmann B (1995) Evidence of contamination in PCR laboratory disposables. Naturwissenschaften 82: 423-431.

32. Neuhuber F, Bettina D, Gabriele H, Jan K, Eva K, et al. (2009) Female criminals - It's not always the offender!. Forensic Sci Int. Genet. Supplement Series 2. 1: 145-146.

33. Daniel R, Oorschot R (2011) An investigation of the presence of DNA on unused laboratory gloves. Forensic Sci Int. Genet. Supplement Series 3: e45-e46. 